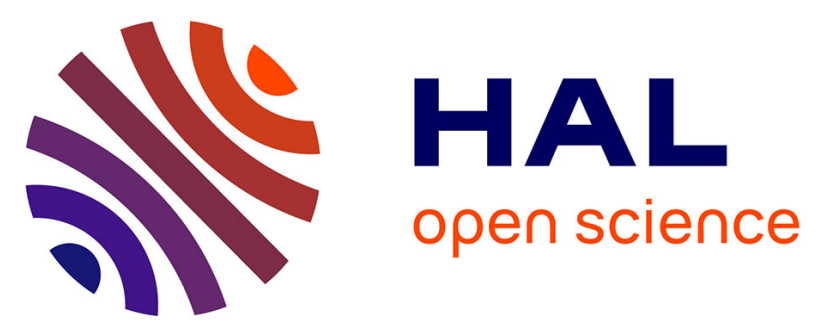

\title{
The histone deacetylase inhibitor vorinostat induces calreticulin exposure in childhood brain tumour cells in vitro
}

Jürgen Sonnemann, Stephanie Gressmann, Sabine Becker, Susan Wittig, Mareike Schmudde, James F. Beck

\section{To cite this version:}

Jürgen Sonnemann, Stephanie Gressmann, Sabine Becker, Susan Wittig, Mareike Schmudde, et al.. The histone deacetylase inhibitor vorinostat induces calreticulin exposure in childhood brain tumour cells in vitro. Cancer Chemotherapy and Pharmacology, 2010, 66 (3), pp.611-616. 10.1007/s00280010-1302-4 . hal-00575248

\section{HAL Id: hal-00575248 \\ https://hal.science/hal-00575248}

Submitted on 10 Mar 2011

HAL is a multi-disciplinary open access archive for the deposit and dissemination of scientific research documents, whether they are published or not. The documents may come from teaching and research institutions in France or abroad, or from public or private research centers.
L'archive ouverte pluridisciplinaire HAL, est destinée au dépôt et à la diffusion de documents scientifiques de niveau recherche, publiés ou non, émanant des établissements d'enseignement et de recherche français ou étrangers, des laboratoires publics ou privés. 


\title{
The histone deacetylase inhibitor vorinostat induces calreticulin exposure in childhood brain tumour cells in vitro
}

\author{
Jürgen Sonnemann · Stephanie Greßmann • \\ Sabine Becker $\cdot$ Susan Wittig $\cdot$ Mareike Schmudde • \\ James F. Beck
}

Received: 9 February 2010 / Accepted: 22 February 2010 / Published online: 10 March 2010

(C) Springer-Verlag 2010

\begin{abstract}
Purpose It has recently been recognised that anticancer chemotherapy can elicit an immunogenic form of apoptosis characterised by the exposure of calreticulin (CRT) on the surface of dying tumour cells, entailing an immune response that contributes to the therapeutic outcome. CRT exposure has been found to be induced by anthracyclins and oxaliplatin, but not by other proapoptotic antineoplastic agents including etoposide, camptothecin and cisplatin. In this study, we examined the histone deacetylase inhibitor vorinostat for its capability to stimulate CRT exposure in tumour cells.

Methods Childhood tumour cells, i.e. the brain tumour cell lines PFSK and DAOY and the Ewing's sarcoma cell line CADO-ES-1, were treated with vorinostat, and CRT exposure was determined by flow cytometric analysis of CRT immunofluorescence. Combination effects of vorinostat/TRAIL and vorinostat/bortezomib were also assessed.

Results Vorinostat treatment induced CRT exposure in PFSK and DAOY cells, but not in caspase-8-deficient CADO-ES-1 cells. CRT exposure could be prevented by the pan-caspase inhibitor z-VAD-fmk and by brefeldin A, an inhibitor of Golgi-mediated transport.
\end{abstract}

J. Sonnemann · S. Greßmann · S. Becker · S. Wittig · J. F. Beck

Department of Paediatric Haematology and Oncology,

University Children's Hospital Jena, Jena, Germany

M. Schmudde

Institute for Immunology and Transfusion Medicine,

Ernst Moritz Arndt University, Greifswald, Germany

J. Sonnemann $(\bowtie)$

Klinik für Kinder- und Jugendmedizin,

Friedrich-Schiller-Universität Jena, Kochstr. 2,

07745 Jena, Germany

e-mail: juergen.sonnemann@med.uni-jena.de
Conclusion Vorinostat has the capacity to elicit CRT exposure, suggesting its usefulness as immunogenic antitumour agent.

Keywords Bortezomib - Calreticulin - Childhood brain tumour · Histone deacetylase inhibitor · TRAIL · Vorinostat

\author{
Abbreviations \\ CRT Calreticulin \\ ER Endoplasmatic reticulum \\ HDACi Histone deacetylase inhibitor \\ sPNET Supratentorial primitive neuroectodermal tumour
}

\section{Introduction}

Histone deacetylase inhibitors (HDACi), such as vorinostat (suberoylanilide hydroxamic acid, SAHA), are an emerging class of promising new anticancer agents. They induce apoptosis, growth arrest and differentiation in tumour cells while leaving healthy cells relatively unscathed [1]. Their clinical potential is underscored by the fact that vorinostat has been approved by the US Food and Drug Administration for treatment of cutaneous T-cell lymphoma [2].

Engagement of antitumour immunity is another promising option to improve the management of malignant disease [3]. Although antineoplastic chemotherapy has long been regarded to induce an immunologically silent or even immunosuppressive response, recent evidence suggests that chemotherapeutic drugs can in fact have immunostimulatory properties. For example, the proteasome inhibitor bortezomib promotes the exposure of heat shock protein 90 on the surface of dying myeloma cells, which leads to the maturation of dendritic cells allowing the latter to activate $\mathrm{T}$ cells [4]. 
Calreticulin (CRT) is another protein whose exposure on the plasma membrane of apoptotic cells can result in the activation of immune cells [5, 6]. CRT usually resides in the endoplasmic reticulum (ER); chemotherapy, however, may cause apoptotic cancer cells to translocate CRT to their surface. The presence of CRT on the surface of tumour cells then directs their uptake by dendritic cells that induce an antitumour immune response [6]. But among the cytotoxic agents tested, only few-the anthracyclins and oxaliplatin-induce CRT translocation and in turn immunogenic cell death, whereas others-e.g. mitomycin C, etoposide, camptothecin and cisplatin—do not [6,7].

HDACi can potentially also stimulate an anticancer immune response. For instance, they have been shown to induce tumour cell-surface expression of NKG2D ligands and boost tumour cell recognition and lysis by NK cells [8-10], to upregulate the expression of MHC class I and class II proteins [11] as well as of costimulatory molecules [12], and to enhance antigen processing and presentation [13]. However, it has not yet been studied whether HDACi cause CRT translocation and, this way, an immunogenic activity.

To explore this issue, we investigated whether vorinostat could induce CRT exposure in childhood brain tumour cells. Brain tumours are the most common solid malignancies of childhood and a leading cause of cancer-related death in children. Despite advances in therapy of childhood brain tumours in general over the last few decades, the prognosis remains dismal for a subset of patients, particularly those with disseminated disease [14]. In addition, current therapies are still frequently associated with severe long-term sequelae. Thus, the development of treatment strategies that may improve the chance of survival as well as lessen side effects is of paramount importance. The utilisation of the immune system to target brain tumours may be a valuable option [15].

We examined a possible CRT-exposing activity of vorinostat in two paediatric brain tumour cell lines: the supratentorial primitive neuroectodermal tumour (sPNET) cell line PFSK and the medulloblastoma cell line DAOY. We have recently shown that vorinostat induces apoptosis in these cell lines $[16,17]$. In the present report, we show that vorinostat treatment also elicits CRT exposure.

\section{Materials and methods}

\section{Reagents}

Vorinostat, mitomycin $\mathrm{C}$, brefeldin $\mathrm{A}$ and $\mathrm{z}-\mathrm{VAD}$-fmk were purchased from Alexis (Grünberg, Germany). Bortezomib was purchased from LC Laboratories (Woburn, MA, USA). TRAIL was purchased from Peprotech (Hamburg, Germany).

\section{Cell lines}

DAOY and PFSK cells were a gift from Dr. M. Grotzer (Zurich, Switzerland) and maintained in Improved MEM Zinc Option (Invitrogen, Karlsruhe, Germany) or RPMI 1640 (PAA, Cölbe, Germany), respectively. CADO-ES-1 cells were obtained from the DSMZ (Braunschweig, Germany) and maintained in RPMI 1640. Media were supplemented with $10 \%$ foetal calf serum, $2 \mathrm{mM}$ L-glutamine, $100 \mathrm{units} / \mathrm{ml}$ penicillin $\mathrm{G}$ sodium and $100 \mu \mathrm{g} / \mathrm{ml}$ streptomycin sulphate (PAA). CADO-ES-1 cells were cultivated in collagen-coated ( $5 \mu \mathrm{g} / \mathrm{cm}^{2}$; Roche, Mannheim, Germany) tissue culture flasks. Cells were cultivated at $37^{\circ} \mathrm{C}$ in a humidified $5 \% \mathrm{CO}_{2}$ incubator and routinely passaged when $90 \%$ confluent. Cell viability was determined by the trypan blue exclusion test. Cells were regularly inspected to be free of mycoplasma with the PCR mycoplasma detection kit from Applichem (Darmstadt, Germany).

\section{Flow cytometric detection of cell-surface CRT}

The cells were plated at $1 \times 10^{5}$ cells in 12 -well plates and treated with vorinostat for $24 \mathrm{~h}$. Brefeldin A or z-VAD-fmk was applied $1 \mathrm{~h}$ before treatment; in the combination experiments, vorinostat was applied $4 \mathrm{~h}$ before TRAIL or bortezomib. Cells were harvested with $0.2 \%$ EDTA, washed twice with PBS, and incubated with the mouse anti-CRT monoclonal antibody FMC $75(2 \mu \mathrm{g}$ in $100 \mu \mathrm{l} \mathrm{PBS} / 2.5 \%$ BSA; Abcam, Cambridge, UK) for $45 \mathrm{~min}$ at $4^{\circ} \mathrm{C}$ in the dark. Cells were washed twice and stained with a FITCconjugated goat anti-mouse IgG antibody $(20 \mu \mathrm{g} / \mathrm{ml}$ in $2.5 \%$ BSA; Dianova, Hamburg, Germany) for $45 \mathrm{~min}$ at $4{ }^{\circ} \mathrm{C}$ in the dark. After washing, 50,000 cells were analysed using a BD (Heidelberg, Germany) FACSCanto II; cells were gated on viable cells. A mouse IgG $(20 \mu \mathrm{g} / \mathrm{ml}$ in $2.5 \%$ BSA; eBioscience, San Diego, CA, USA) was used as isotype-matched control. Frequency histograms were generated using Flowjo software (Ashland, OR, USA). Data are shown as fold induction after calculation of the mean fluorescence intensities of anti-CRT-stained cells divided by isotype-matched IgG-stained cells.

Immunoblot detection of total CRT

After a 24-h incubation with vorinostat, cells were lysed on ice for $15 \mathrm{~min}$ in $40 \mathrm{mM}$ Tris- $\mathrm{HCl}$ (pH 7.4), $150 \mathrm{mM} \mathrm{NaCl}$, $1 \%$ Triton $\mathrm{X}-100,0.5 \%$ sodium deoxycholate and $0.1 \%$ SDS supplemented with a protease inhibitor cocktail (Roche) followed by brief sonification. Protein concentration was assayed using bicinchoninic acid (Pierce, Rockford, IL, USA) according to the manufacturer's instructions. Concentration of $30 \mu \mathrm{g}$ of total cellular protein per lane was separated by standard SDS-PAGE on $10 \%$ gel and 
electrophoretically transferred to nitrocellulose membrane (Whatman, Dassel, Germany). After blocking in PBS containing $5 \%$ dry milk and $0.05 \%$ Tween 20 , CRT was immunodetected using FMC 75 antibody (dilution 1:1,000). Equal loading of protein was verified by detection of GAPDH using mouse anti-GAPDH monoclonal antibody (dilution 1:10,000; Biodesign International, Saco, ME, USA). Peroxidase-conjugated goat anti-mouse IgG (dilution 1:25,000; Dianova) was used as secondary antibody followed by enhanced chemiluminescence detection (Amersham, Freiburg, Germany) of specific signals.

\section{Statistical analysis}

Statistical significance of differences between experimental groups was determined using the paired two-tailed Student's $t$ test.

\section{Results}

\section{CRT exposure on PFSK sPNET cells}

To test whether vorinostat elicits CRT exposure in paediatric brain tumour cells, we initially determined CRT surface expression on PFSK cells. Figure 1a, b demonstrates that vorinostat induced CRT exposure in a dose-dependent manner, as monitored by flow cytometric analysis of viable cells for CRT immunofluorescence staining. To exclude a passive leakage of CRT to the plasma membrane, cells were pretreated with brefeldin $\mathrm{A}$, an inhibitor of protein transport from the ER to the Golgi apparatus: Inhibition of ER-Golgi traffic abolished vorinostat-elicited CRT exposure. Because chemotherapy-mediated CRT translocation has been shown to involve caspases [6], PFSK cells were pretreated with the pan-caspase inhibitor z-VAD-fmk: Caspase inhibition prevented the CRT-exposing activity of vorinostat.

\section{CRT exposure on DAOY medulloblastoma cells}

Next, we studied the effect of vorinostat on CRT exposure in DAOY cells. As shown in Fig. 1c, vorinostat treatment also provoked a concentration-dependent CRT surface expression in these cells. As a negative control, DAOY cells were also treated with mitomycin $\mathrm{C}$, which has been reported to exert no CRT-exposing effect [6]. Mitomycin C did not increase CRT exposure in DAOY cells. In addition, we examined whether CRT exposure in vorinostat-treated cells was accompanied by a general rise of CRT abundance. Immunoblot detection of CRT in total cell lysates showed that vorinostat treatment did not result in an increase in total CRT (Fig. 1d).
CRT exposure on CADO-ES-1 Ewing's sarcoma cells

Caspase- 8 has been shown to be necessary for anthracyclinand oxaliplatin-induced CRT exposure [18]. To shed light on the relevance of caspase- 8 for HDACi-induced CRT exposure, we employed the Ewing's sarcoma cell line CADO-ES-1, a cell line deficient in caspase-8 expression [19], but apoptotically responsive to HDACi treatment [20]. Figure 1e illustrates that vorinostat had no effect on CRT surface expression on CADO-ES-1 cells.

CRT exposure after vorinostat/TRAIL or vorinostat/bortezomib combination treatment

A large number of preclinical studies have demonstrated synergistic proapoptotic activity of vorinostat with other antineoplastic agents, such as the death receptor ligand TRAIL or the proteasome inhibitor bortezomib [2]. We thus evaluated whether vorinostat could also synergise with TRAIL or bortezomib in triggering CRT exposure. In consistence with the observation that TRAIL elicits CRT exposure in Hela cells [18], we found that TRAIL evoked CRT surface expression on DAOY cells (Fig. 2a). Yet the vorinostat/TRAIL combination treatment produced a merely additive effect. However, when vorinostat was combined with bortezomib, a trend to overadditive induction of CRT exposure was observed (Fig. 2b).

\section{Discussion}

To the best of our knowledge, we herein report for the first time that treatment with an HDACi leads to CRT surface exposition on tumour cells. Given the limited number of chemotherapeutics with proven CRT-exposing activitythe anthracyclins and oxaliplatin $[6,7]-$ this entails a substantial expansion of antineoplastic agents endowed with the potential to elicit a CRT-dependent antitumour immune response.

In our study, we have shown that vorinostat induced CRT exposure in a concentration-dependent fashion. Several lines of evidence presented indicate that vorinostat promotes CRT exposure by the same mechanism as the known CRT-exposing compounds. First, we could not detect a rise of total CRT expression, in concordance with the finding that exposure of CRT induced by anthracyclins does not stem from a general increase in CRT abundance [6]. Second, CRT translocation has been demonstrated to occur via the ER-Golgi pathway [18]. Our results show that the vorinostat-mediated CRT exposure could be prevented by brefeldin A, suggesting that CRT translocation induced by vorinostat also depends on anterograde ER-Golgi traffic. Third, in line with the observation that chemotherapy-elicited 
Fig. 1 Induction of CRT exposure by vorinostat. Cells were treated with vorinostat for $24 \mathrm{~h}$. $\mathbf{a}, \mathbf{b}, \mathbf{c}, \mathbf{e}, \mathrm{CRT}$ cell-surface expression was determined by flow cytometric analysis of viable cells. a, Cells were stained with CRT antibody (solid line) or isotype control (filled histogram). b, c, e, CRT mean fluorescence is depicted as fold induction after calculation of the mean fluorescence intensities of anti-CRT-stained cells divided by isotype-matched IgG-stained cells; mean \pm SEM. of each 3 experiments are shown (*treatment vs. control; " brefeldin A and z-VAD-fmk, respectively, vs. $5 \mu \mathrm{M}$ vorinostat). d, total CRT expression was determined by immunoblot analysis of cell lysates

\section{PFSK}

A
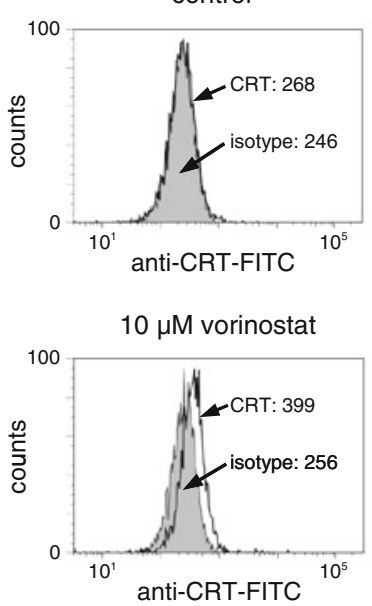

B

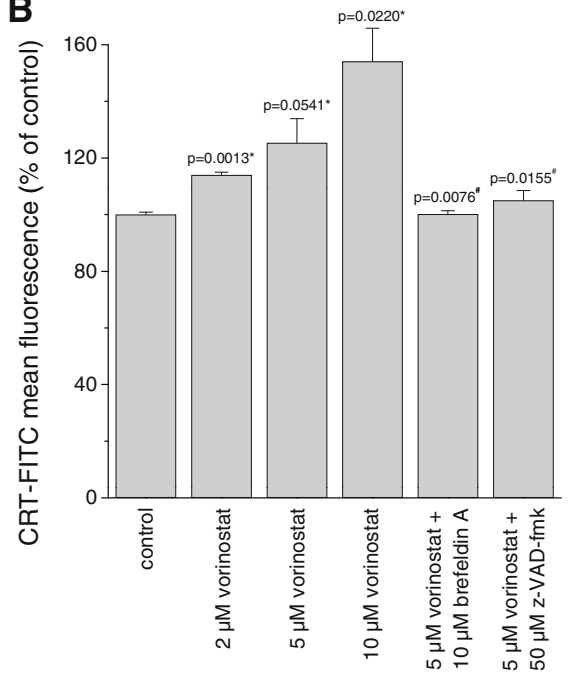

\section{C}

\section{DAOY}

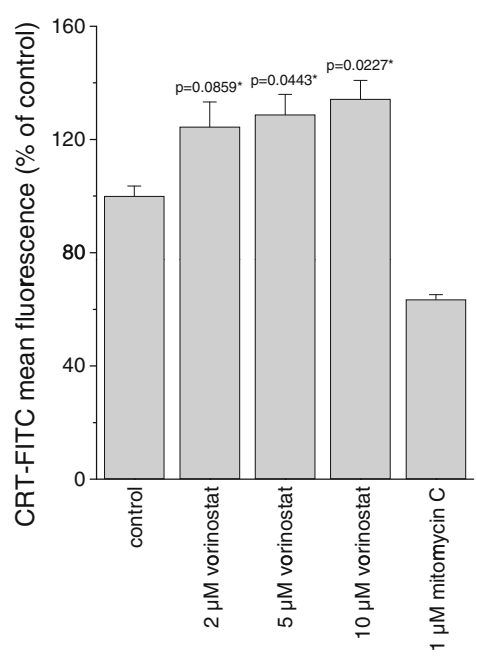

D

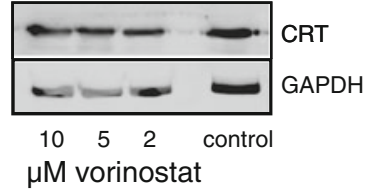

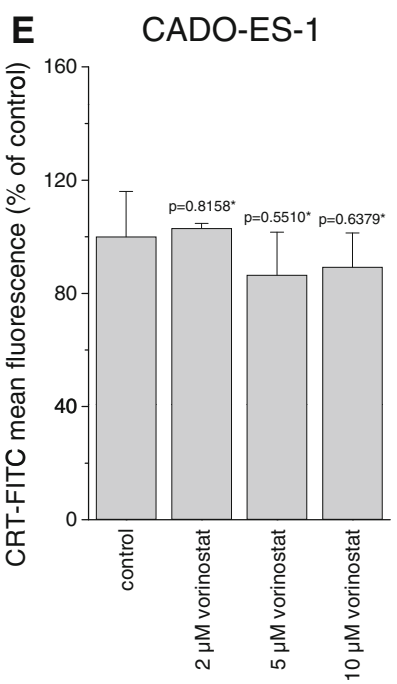


Fig. 2 Induction of CRT exposure by vorinostat in combination with TRAIL or bortezomib. $4 \mathrm{~h}$ after administration of vorinostat, DAOY cells were treated with TRAIL (a) or bortezomib (b) for $24 \mathrm{~h}$. CRT cell-surface expression was determined by flow cytometric analysis of viable cells. CRT mean fluorescence is depicted as fold induction after calculation of the mean fluorescence intensities of anti-CRT-stained cells divided by isotype-matched IgGstained cells; mean \pm SEM. of each 3 experiments are shown $(*$ treatment vs. control; \# combination treatment vs. vorinostat; ${ }^{\S}$ combination treatment vs. TRAIL or bortezomib, respectively)

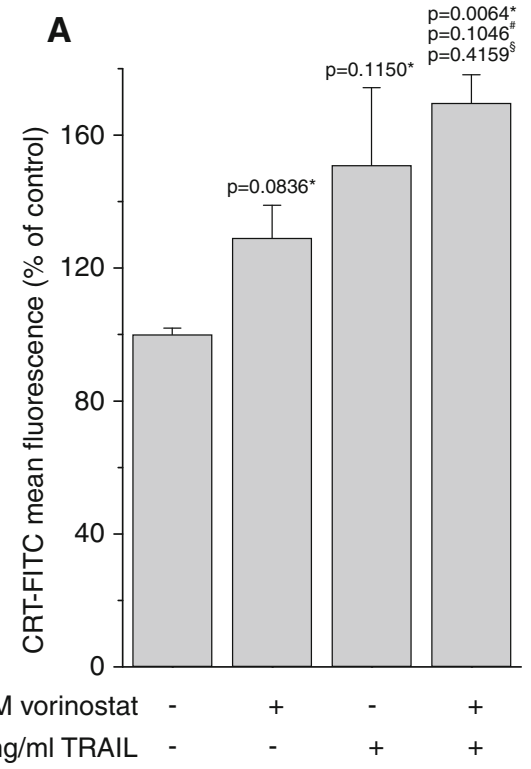

CRT surface expression depends on caspase activity [6], vorinostat-induced CRT exposure could be abolished by z-VAD-fmk. In addition, vorinostat failed to promote CRT exposure in caspase-8-deficient CADO-ES-1 cellswhich are otherwise susceptible to HDACi-treatment [20] - consistent with the finding that caspase- 8 is necessary for CRT exposure, but not apoptosis, induced by anthracyclins and oxaliplatin [18].

A multitude of preclinical studies have documented that HDACi have great potential to augment the anticancer efficiency of other therapeutic regimens, such as chemoand radiotherapy $[1,2]$. With respect to the use of TRAIL for the treatment of childhood tumours, we have previously shown that vorinostat and TRAIL synergise to elicit cell death in medulloblastoma, Ewing's sarcoma and hepatoblastoma [17, 20, 21]. TRAIL seemed furthermore to be particularly interesting since it has been demonstrated to stimulate CRT exposure in carcinoma cells [18]. In the attempt to find out whether vorinostat and TRAIL synergised in inducing CRT exposure, however, we detected a merely additive effect. In contrast, the combination of vorinostat with bortezomib - the latter has been reported to elicit immunogenic cell death by inducing exposure of heat shock protein 90 [4] — produced a trend to enhanced induction of CRT surface expression. These findings show that the combination of two CRT-exposing compounds does not necessarily result in the potentiation of CRT exposure, but they also show that it can in principle be augmented by a suitable combination treatment.

Again to the best of our knowledge, we here have also shown for the first time that childhood brain tumour cells can respond to antineoplastic agents by exposing CRT. Therefore, our results suggest that the concept of immunochemotherapy [3] may be a therapeutic option in these difficult to treat tumours. Chemotherapy of brain tumours is in general complicated in that the blood-brain barrier obstructs the penetration of most drugs into the brain [22]. However, this shortcoming does supposedly not apply to vorinostat, for it has been shown to cross the blood-brain barrier in mice [23]. To sum up, our study on the one hand extends the notion that HDACi have immunomodulatory activity that may contribute to mediating their antitumour effects [8-13] and on the other supports their potential usefulness in paediatric oncology [24].

Acknowledgments We gratefully acknowledge the input and discussions of Barbara Bröker (Institute for Immunology, University of Greifswald). This work was supported by a grant from the "Wilhelm Sander-Stiftung, Neustadt/Donau". S.G. received a fellowship from the "IZKF des Universitätsklinikum Jena"; M.S. received a fellowship from the "Alfried Krupp Wissenschaftskolleg Greifswald der Alfried Krupp von Bohlen und Halbach-Stiftung".

\section{References}

1. Lane AA, Chabner BA (2009) Histone deacetylase inhibitors in cancer therapy. J Clin Oncol 27:5459-5468

2. Richon VM, Garcia-Vargas J, Hardwick JS (2009) Development of vorinostat: current applications and future perspectives for cancer therapy. Cancer Lett 280:201-210

3. Zitvogel L, Apetoh L, Ghiringhelli F, Andre F, Tesniere A, Kroemer G (2008) The anticancer immune response: indispensable for therapeutic success? J Clin Invest 118:1991-2001

4. Spisek R, Charalambous A, Mazumder A, Vesole DH, Jagannath S, Dhodapkar MV (2007) Bortezomib enhances dendritic cell (DC)-mediated induction of immunity to human myeloma via exposure of cell surface heat shock protein 90 on dying tumor cells: therapeutic implications. Blood 109:4839-4845

5. Gardai SJ, McPhillips KA, Frasch SC et al (2005) Cell-surface calreticulin initiates clearance of viable or apoptotic cells through trans-activation of LRP on the phagocyte. Cell $123: 321-334$ 
6. Obeid M, Tesniere A, Ghiringhelli F et al (2007) Calreticulin exposure dictates the immunogenicity of cancer cell death. Nat Med 13:54-61

7. Tesniere A, Schlemmer F, Boige V et al (2010) Immunogenic death of colon cancer cells treated with oxaliplatin. Oncogene 29:482-491

8. Armeanu S, Bitzer M, Lauer UM et al (2005) Natural killer cellmediated lysis of hepatoma cells via specific induction of NKG2D ligands by the histone deacetylase inhibitor sodium valproate. Cancer Res 65:6321-6329

9. Skov S, Pedersen MT, Andresen L, Straten PT, Woetmann A, Odum N (2005) Cancer cells become susceptible to natural killer cell killing after exposure to histone deacetylase inhibitors due to glycogen synthase kinase-3-dependent expression of MHC class I-related chain A and B. Cancer Res 65:11136-11145

10. Schmudde M, Braun A, Pende D et al (2008) Histone deacetylase inhibitors sensitize tumour cells for cytotoxic effects of natural killer cells. Cancer Lett 272:110-121

11. Magner WJ, Kazim AL, Stewart C et al (2000) Activation of MHC class I, II, and CD40 gene expression by histone deacetylase inhibitors. J Immunol 165:7017-7024

12. Maeda T, Towatari M, Kosugi H, Saito H (2000) Up-regulation of costimulatory/adhesion molecules by histone deacetylase inhibitors in acute myeloid leukemia cells. Blood 96:3847-3856

13. Setiadi AF, Omilusik K, David MD et al (2008) Epigenetic enhancement of antigen processing and presentation promotes immune recognition of tumors. Cancer Res 68:9601-9607

14. Packer RJ (2008) Childhood brain tumors: accomplishments and ongoing challenges. J Child Neurol 23:1122-1127

15. Mitchell DA, Fecci PE, Sampson JH (2008) Immunotherapy of malignant brain tumors. Immunol Rev 222:70-100
16. Kumar KS, Sonnemann J, Beck JF (2006) Histone deacetylase inhibitors induce cell death in supratentorial primitive neuroectodermal tumor cells. Oncol Rep 16:1047-1052

17. Sonnemann J, Kumar KS, Heesch S et al (2006) Histone deacetylase inhibitors induce cell death and enhance the susceptibility to ionizing radiation, etoposide, and TRAIL in medulloblastoma cells. Int J Oncol 28:755-766

18. Panaretakis T, Kepp O, Brockmeier U et al (2009) Mechanisms of pre-apoptotic calreticulin exposure in immunogenic cell death. EMBO J 28:578-590

19. Fulda S, Kufer MU, Meyer E, van Valen F, Dockhorn-Dworniczak B, Debatin KM (2001) Sensitization for death receptor- or drug-induced apoptosis by re-expression of caspase- 8 through demethylation or gene transfer. Oncogene 20:5865-5877

20. Sonnemann J, Dreyer L, Hartwig M et al (2007) Histone deacetylase inhibitors induce cell death and enhance the apoptosis-inducing activity of TRAIL in Ewing's sarcoma cells. J Cancer Res Clin Oncol 133:847-858

21. Dzieran J, Beck JF, Sonnemann J (2008) Differential responsiveness of human hepatoma cells versus normal hepatocytes to TRAIL in combination with either histone deacetylase inhibitors or conventional cytostatics. Cancer Sci 99:1685-1692

22. Muldoon LL, Soussain C, Jahnke K et al (2007) Chemotherapy delivery issues in central nervous system malignancy: a reality check. J Clin Oncol 25:2295-2305

23. Hockly E, Richon VM, Woodman B et al (2003) Suberoylanilide hydroxamic acid, a histone deacetylase inhibitor, ameliorates motor deficits in a mouse model of Huntington's disease. Proc Natl Acad Sci USA 100:2041-2046

24. Fruhwald MC, Witt O (2008) The epigenetics of cancer in children. Klin Padiatr 220:333-341 